First Peoples Child \& Family Review

A Journal on Innovation and Best Practices in Aboriginal Child Welfare Administration,

Research, Policy \& Practice

\title{
Fetal Alcohol Spectrum Disorder Standards: Supporting Children in the Care of Children's Services
}

\section{Dorothy Badry}

Volume 4, Number 1, 2009

URI: https://id.erudit.org/iderudit/1069349ar

DOI: https://doi.org/10.7202/1069349ar

See table of contents

Publisher(s)

First Nations Child and Family Caring Society of Canada

\section{ISSN}

1708-489X (print)

2293-6610 (digital)

Explore this journal

Cite this article

Badry, D. (2009). Fetal Alcohol Spectrum Disorder Standards: Supporting

Children in the Care of Children's Services. First Peoples Child \& Family Review, 4(1), 47-56. https://doi.org/10.7202/1069349ar

\section{Article abstract}

The purpose of this research was to examine the utilization of enhanced practice standards for children in care with Fetal Alcohol Spectrum Disorder (FASD). Children in care with FASD represent a vulnerable population and require multiple supports from a cross-disciplinary perspective. Children removed from the care of their parents were identified as having needs beyond standard care provided within Children's Services in Alberta. To address this concern a project was initiated in 2002 and completed in 2005 which identified positive benefit from an increase in caseload hours for workers responsible for children with FASD in the Aboriginal Unit including more contact with children and additional supports for foster parents. Standards regarding family visitation are also highlighted. An additional casework position was developed in order to decrease caseloads and meet the standards. Changing the way child welfare and foster care services are delivered for children with FASD is an important phenomenon to study and this research may guide future interventions.
This document is protected by copyright law. Use of the services of Érudit (including reproduction) is subject to its terms and conditions, which can be viewed online.

https://apropos.erudit.org/en/users/policy-on-use/ 


\title{
Fetal Alcohol Spectrum Disorder Standards: Supporting Children in the Care of Children's Services
}

\author{
Dorothy Badrya
}

${ }^{\text {a }} \mathrm{PhD}, \mathrm{RSW}$, Faculty of Social Work, University of Calgary, Calgary, Alberta, Canada

\section{Introduction}

Fetal Alcohol Spectrum Disorder (FASD) is a complex phenomenon in our society. Although identified as preventable, the issue of alcohol use during pregnancy and the consequence of children diagnosed with FASD require extensive interventions with women with traumatic histories to support prevention. Often, children who are raised in homes where alcohol is a problem end up in the care of the state. The focus of this research was to evaluate FASD Practice Standards for children in the care of Alberta Children's Services. This research offered the opportunity to examine the issues and efforts in child welfare practice to address concerns for children, families and caregivers living with FASD.

Hutson (2006) estimates that "3000 babies are born with FASD in Canada" and cites a report from the Child Welfare League of Canada (2003) which further estimates "that $50 \%$ of children in care in Alberta have FAS" (p. 2). Alberta Health Services suggests that it is estimated that 9 of every 1000 births has a fetal alcohol spectrum disorder. Fuchs, Burnside, Marchenski and Mudry (2005) in their research of children in the care of child welfare agencies in Manitoba estimated that $17 \%$ of children in care have a fetal alcohol spectrum disorder. Variance amongst prevalence

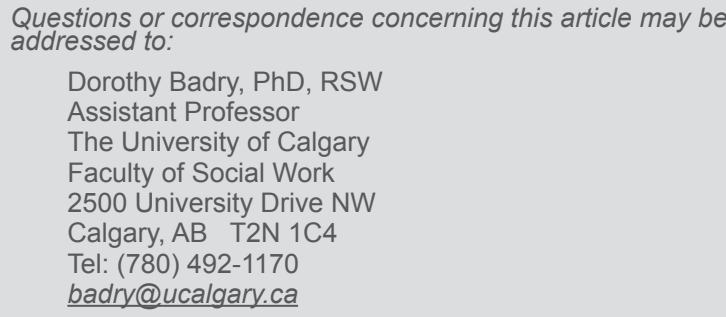

\begin{abstract}
The purpose of this research was to examine the utilization of enhanced practice standards for children in care with Fetal Alcohol Spectrum Disorder (FASD). Children in care with FASD represent a vulnerable population and require multiple supports from a cross-disciplinary perspective. Children removed from the care of their parents were identified as having needs beyond standard care provided within Children's Services in Alberta. To address this concern a project was initiated in 2002 and completed in 2005 which identified positive benefit from an increase in caseload hours for workers responsible for children with FASD in the Aboriginal Unit including more contact with children and additional supports for foster parents. Standards regarding family visitation are also highlighted. An additional casework position was developed in order to decrease caseloads and meet the standards. Changing the way child welfare and foster care services are delivered for children with FASD is an important phenomenon to study and this research may guide future interventions.
\end{abstract}

rates exist as there is not a coordinated approach in Canada to gathering this information.

\section{Origins of the Development of FASD Practice Standards}

In 2001, a group of community stakeholders and child welfare professionals met in Lethbridge, Alberta and collaboratively agreed that children in care with Fetal Alcohol Syndrome (FAS) / Fetal Alcohol Spectrum Disorder (FASD) were not being adequately served by existing standards of care. The major reason behind the decision to examine this problem was the concern about the complex needs of children with FASD primarily due to disabilities resulting from alcohol exposure in-utero. This led to the design of practice standards specific to the identified needs of children in 2002. The standards directly related to child welfare intervention included: screening for FASD, child assessment, parenting ability, case plans, case plan review, home visits, case worker contact, child and 
family awareness of FASD and permanency planning. Key standards related to management of the project addressed training for staff and caregivers, assessments for youth and parents, support for foster parents, kinship care, transition to adulthood, case worker contact and case plan reviews.

Once the standards were developed, Region 1 of Alberta Children's Services contacted the Faculty of Social Work, University of Calgary to request assistance in evaluation of this project. Funding for this research came from the Alberta Centre for Child, Family and Community Research (ACCFCR) in 2003. The standards were field tested in 2002 and fine tuned and this pilot research project ran from 2003-2005. An extensive final report was completed in 2005 (Badry, Pelech and Norman). The full report is located online on the FASD Connections Website (http:// www.fasdconnections.ca/id112.htm).

The initial challenge of this research was that no prior published research literature existed regarding the care of children with FASD who were in the care of the state. Social science research has not kept pace with biomedical research in relation to FASD and this posed a challenge in terms of research direction and design. Streissguth (1997) and Streissguth and Kanter (1997) published seminal research in terms of not only the medical conditions associated with FASD, but the profound social/behavioral/ familial problems associated with a diagnosis. A host of neurobehavioral, psychosocial and health issues seemed inherent with a diagnosis of FASD.

In presenting this research it is critical to highlight important issues relevant to child protection. This paper represents the FASD Practice Standards research as well as some philosophical considerations in the delivery of services to children with FASD. Having worked for 16 years in child welfare (1986-2002) I had the opportunity to witness the growing awareness of FASD as a concern in child welfare practice. My interest in research related to FASD emerged from the opportunity to work with a 16 year old youth in 1986 whose mother was alcoholic. He had visible characteristics of FAS but diagnostic clinics did not exist at the time. He told me he had problems because his mother drank while pregnant with him. I never met his mother as he was an emancipated youth. Today, this young man would likely have access to a diagnostic clinic and subsequent supports for children with FASD that were not available 20 years ago. This signals progress in our understanding of FASD but there is still a long way to go in developing practice models. The FASD Practice Standards research signals a beginning.

\section{Brief History of Fetal Alcohol Syndrome / Fetal Alcohol Spectrum Disorder}

The medical system opened the social discourse on FAS by raising the issue in France (Lemoince, 1968) and by Jones, Smith, Ulleland and Streissguth (1973) in North America. These researchers recognized that a cluster of children existed with similar physical features and medical problems, and recognized the common linkage between these characteristics was the teratogenic or toxic effects of alcohol on the developing fetus. They named this condition, Fetal Alcohol Syndrome (FAS). The term FAS describes a myriad of features, both physical and neurological. It does not address the social dimensions and consequences related to the phenomenon of living with the condition. The medical system has become the primary informant about this topic as a public health issue. The scientific study of the teratogenic effects of alcohol on the developing fetus has evolved into a complex field of medical assessment and diagnosis, and FAS has been identified as a topic of great concern to society.

Recent literature offers the term FetalAlcohol Spectrum Disorders, as a term that refers to the many conditions that can result from prenatal alcohol exposure (Streissguth and O'Malley, 2001). The term FAS was frequently referred to in the literature that predates 2002-2003. The term FAS has more recently become housed by the medical profession under a broader description, known as Fetal Alcohol Spectrum Disorder (FASD) (Streissguth and O'Malley, 2000). The medical profession has indicated that multiple conditions exist along the spectrum of potential disorders resulting from alcohol exposure in utero and utilizing an umbrella term promotes common understanding that the cause of the problems a child lives with are alcohol related (O’ Malley and Streissguth, 2002).

Canadian researchers have developed Canadian Guidelines for Diagnosis (Chudley et al., 2005). While the physical etiology of FAS / FASD was well documented, the social etiology slowly unfolded as awareness grew of the profound impact of alcohol exposure on human development. In particular, Streissguth (1997) was a pivotal researcher in bringing awareness to the psychosocial issues related to a diagnosis of FAS (Streissguth, 1997, Streissguth and Kanter, 1997) and FASD (Streissguth and O’Malley, 2001).

\section{A Moralized Discourse Underlies Complex Social Issues}

If the term 'prenatal substance abuse' is used, a woman is immediately implicated as guilty and placed within a dichotomous framework where harm / control of her body is weighed against harm / control to the fetus. A less loaded term to utilize instead is alcohol exposure in utero, to diminish stigma. Framing the issue of FASD from a child protection standpoint immediately places a mother in conflict with this system. The welfare of children is considered to be a public issue. One of the roles of women as mothers is to protect children from harm. Through a societal lens and driven by a dominant medical response to 
this issue, women who drink during pregnancy are causing undue harm to their children. One of the problems with this position is that not all women who drink during pregnancy give birth to children with FAS. However, women who do give birth to children with FAS are perceived as unfit, portrayed as irresponsible and even malevolent.

In reflecting upon the birth of children with FASD, it is important to contextualize the experience of women. Fetal Alcohol Spectrum Disorder, in contemporary Western society, has become overtly represented as a moralized disability. As such, the evolution of a prevailing discourse, grounded in the bio-medical definition of FASD, that suggests if only women refrain, by choice, from alcohol use during pregnancy, then FASD will cease to exist. Discourse on prevention suggests if pregnant women do not refrain from alcohol, then these mothers-to-be must be held responsible - ethically, socially, morally, medically, politically - for any alcohol-related difficulties the child experiences. I raise this issue as my doctoral dissertation was about birth mothers of children with FAS and I sought to humanize their experience through my research. The intergenerational issues of FAS/FASD will only be mediated by protecting all children from physical, sexual, emotional abuse and neglect. The problematic use of alcohol within our society is rarely mentioned in discussions about FASD.

According to Poole (2007) a focus on Aboriginal women based on limited research has resulted in negative assumptions of higher prevalence than other populations. More recently, studies have come forward which provide a critical analysis of the topic of the social construction of knowledge about women who consume alcohol during pregnancy and the language used to describe this topic (Ferguson, 1997; 2003a; Armstrong, 2003; Boyd, 2004; Boyd and Marcellus (2007). For example, Tait, (2000 a2003b) engages in a sociological analysis on The Tip of the Iceberg: The Making of Fetal Alcohol Syndrome in Canada in her doctoral dissertation, suggesting FAS has been constructed as a response to stigmatize the First Nations people of Canada and North America. Berube (2005) represented birth mothers with the metaphor of "modern lepers" due to her perception of the negative societal response to pregnant, alcoholic women. Armstrong and Abel (2000) have flagged a concern about identifying "moral disorder" in women through the diagnosis of their children with FAS (p. 276).

Tait (2003b) stated the following:

Despite the negative impacts of the residential school system and other forms of colonization, it should be pointed out that not every former student responded in the same way to their experience and, for various reasons, some individuals and communities did better than others did. Because of this alcohol abuse among

\begin{abstract}
Aboriginal people in Canada varies and it should be understood as a problem of certain individuals and subpopulations, rather than a problem of all Aboriginal people. In relation to [FAS / FASD], this suggested that programming and services should target those particular populations who are at risk, rather than targeting all groups regardless of the alcohol use levels ( $p . x v$ ).
\end{abstract}

Poole (2009) identifies a child-centred focus as a barrier to "understanding women's substance use in pregnancy and into developing the interventions needed to prevent this use and/or mitigate the harms associated with it" (p. 290). The implications of a diagnosis of FASD are far reaching for both a child and mother. Social science research related to fathers has lagged with only one published study regarding the role of fathers (Gearing, McNeill and Lozier, 2005).

Barr \& Streissguth (2001) raise the issue of maternal self-report in relation to supporting a diagnosis since no standardized test exists to assess the level of alcohol exposure to a fetus. It is not unusual for women to under report their substance use out of fear of consequences such as a referral to child welfare and risk of child apprehension. Streissguth $(1997,2002)$ reported on research occurring as early as 1974-1975 in the Seattle area, on women using drugs and alcohol who were involved with prenatal care. Streissguth suggested that knowledge of alcohol exposure is important in not only identifying those at risk, but can also be a support in early planning and intervention that can mediate against child protection concerns for the family.

Precursors that lead to alcohol use during pregnancy include a history of childhood sexual abuse, and alcoholism within the family of origin (Tait, 2003). Marsh and Dingcai (2005) report that substance abuse is a major factor in the removal of children from parental care and recommend an intervention model which integrates child welfare and substance abuse treatment, in the interest of better outcomes for children. Grella, Hser and Huang (2006) suggest that early intervention with parental substance abuse treatment may "interrupt their substance use prior to progressing to later stages of addiction severity" (p. 69). Multiple measures are critical for a diagnosis of FASD and maternal self-report alone cannot support a diagnosis. There is often discomfort on the part of health or social service professionals around asking about alcohol use in pregnancy. Although information on the consumption of alcohol use during pregnancy is a critical part of a diagnostic assessment for FASD, this is an extremely sensitive issue that must be cautiously addressed. Asking the question of alcohol use during pregnancy in a child protection investigation can traumatize women and further research on this topic is required.

Grant, Ernst, Streissguth and Porter (1997) suggested that many women are reluctant to reveal their use of alcohol 
during pregnancy because of fear of judgment, alienation, and removal of the child from their care. The issue of fathers is rarely mentioned in the literature with only one study emerging which highlights the concern that the influence of fathers has largely been ignored, yet their role is relevant and further research in the area of FASD and the family is required (Gearing, McNeill and Lozier, 2005). Tait (2000a) suggests that relationship problems within families, community and with male partners contribute to alcohol misuse. A holistic view of the problems associated with the diagnosis of a child with FASD may be difficult to establish because families are often fractured through child apprehension and placement in foster care. If the misuse of alcohol and substance abuse are not mediated through treatment and support, the risks to children in the home remain problematic. Streissguth (1997) indicated that birth mothers lives are often "out of control on all fronts" (p. 271), and child protection is often required. As well, intensive in-home supports are required for the birth mother if the apprehended child was to return to parental care.

The concerns of child protection for children of alcoholic mothers are very serious and cannot be minimized. Alcoholism is a serious issue within families but it needs to be contextualized within the historical experience of families. Blaming women as the cause will not support decreasing births of children with FASD. Women who find themselves in this circumstance of becoming birth mothers have complex histories. Perry (2002) identified The Vortex of Violence as the ways in which children adapt to living in violent circumstances. There is an intergenerational cycle in which girls that are abused as children find ways to adapt as they grow up. Perry stated, "Persisting fear and adaptations to the threat present in the vortex of violence... contribute to the transgenerational cycle of violence as these young children become adolescents - and finally, the adults that shape our society" (p. 4). Alcohol use is often a co-morbid factor in violence.

The way parents are treated in child protection matters influences the outcomes for children in care. The way caseworkers interact with parents is critical and standards that guide that practice are imperative in the interests of better outcomes for children and families. One of the realities is that parents themselves may be alcohol exposed and this presents challenges in practice. The complexity of FASD, the need for psychosocial and medical intervention from an interdisciplinary perspective alone suggests that a model of specialized practice is required. As knowledge has grown about FASD the possibility exists to develop new models of practice and interventions that may challenge current policy practice frameworks.
It is important to contextualize the framework which has driven the need to respond to FASD differently by establishing specific casework standards. The issue of child neglect is a concern in families where substance use is a problem. The active alcoholic can experience difficulties in meeting the needs of their children and security in the home environment becomes disrupted. Poverty is also a concern and those children experiencing neglect are more likely to come from homes without full time employment outcome and utilizing other sources such as social assistance, other pensions or part time work (Roy, Black, Trocmé, MacLaurin and Fallon, 2005). Lack of stability creates risk for children with FASD as routine and structure mediate their neurodevelopmental disabilities.

Vig, Chinitz and Shulman (2005) completed a study that examined young children in care with complex needs and multiple vulnerabilities, and suggested that $80 \%$ may have had prenatal exposure to alcohol. Factors cited in removal of children from parental (biological) care broadly includes "neglect" and "parental incapacity" (p. 147). Aronson, (in Streissguth \& Kanter,1997) suggests that "even though early fostering did not appear to eliminate the harmful effects of exposure to alcohol in utero, foster care seems to be the most favorable alternative for children whose biological mothers, despite vigorous attempts at psychological support, continue to abuse alcohol and have severe personal psychological problems. Children prenatally exposed to alcohol who remain in biological families....remain at continued risk" (p. 24). For children with FASD, stability and structure are hallmarks of good care.

A phenomenon of concern that has been addressed in the literature by Streissguth (1997); Grant, Ernst, Streissguth and Stark (2005) is the lives of birth mothers. There are not many opportunities to raise the voices of birth mothers which suggest they are a disenfranchised population. It is important that the concerns of birth mothers be raised in the discourse because it will help deepen an understanding of families where child welfare engages in protection/ apprehension interventions. Some of the characteristics from my own qualitative research on eight birth mothers, primarily non-Aboriginal was that they were often single mothers, had unplanned pregnancies, engaged in transient relationships with others, had a history themselves of child welfare involvement, often combined alcohol and drug use and were exposed to alcoholism in their families of origin. This leads to women with fragile histories becoming invisible to the system once their children are apprehended due to their difficulties in trusting others and viewing systems such as child welfare as a threat rather than a support (Badry, 2009).

The reason it is important to highlight the concerns of

\section{Child Protection Framework}


the mother is that interventions in child welfare focus on the child and not on the needs of the mother. Perhaps a deeper understanding of the history of birth mothers who themselves often have histories of abuse and neglect can lead to a more compassionate response for birth mothers and guide interventions related to family functioning. An important concern is the possibility that the birth mother may have been alcohol exposed which has implications for finding ways to work effectively with families and designing accommodations in service delivery for both mother and child, perhaps decreasing the need for apprehensions. This concern also leads to the question of adult diagnosis which is currently a topic of interest in Alberta (Clarren and Lutke, 2009). Knowledge about birth mothers may help child welfare to better understand women's issues related to parenting. Additionally, intergenerational issues will not be resolved if the healing process excludes rather than includes the family. This topic warrants further exploration but is beyond the scope of the current research.

Another concern for children is the importance of living in an environment where they have the opportunity to develop secure attachments with immediate and extended family wherever possible. With active substance misuse, secure attachment is a concern for infants and young children. Disrupted attachment is a consequence of substance misuse due to its disruption of the nurturing parent/ child relationship. Concern for children with FASD around their ability to attach is heightened when they experience multiple disruptions in life. "With each additional disruption, the ability to bond diminishes" (Alberta Children's Services Workforce Development and Gough, 2007, p.1). When child protection needs are serious children are often removed from their home and placed in foster care or kinship care. In an analysis of issues in Canada related to the placement of Aboriginal children in care, Gough, Trocme', Brown, Knoke and Blackstock (2005) identify a disproportionate representation of these children in care. In particular, Gough et al. (2005) note that the numbers continue to rise despite the awareness of this concern and indicate that the placement of Aboriginal children in care is a critical question requiring ongoing discourse and research.

\section{Cascading Vulnerabilities}

Children with FASD have cascading vulnerabilities physically, emotionally, mentally and spiritually when they are not offered adequate supports within systems that are designed to protect them and provide developmental stability. When I searched the words "cascading vulnerabilities", a term which emerged from my reflections and research regarding children with FASD and their many struggles, I discovered this was an engineering term. The original meaning of this term relates to the potential of large scale failure of electrical systems when particular parts of the system are disrupted. The loss of power in one part of the system results in loss in other parts of the system which continue to build into large scale blackouts across the grid. This metaphor represents a challenge to develop models of practice that recognize the impact of loss for children with FASD and the need to build strong systems that will not fail them in their profound need for security and developmental stability whether at home or in care.

Children with FASD can have particular physical vulnerabilities such as developmental delays, neurobiological problems, physical problems such as heart disease, musculoskeletal problems and compromised immune systems (Stratton, Battaglia and Howe, 1996). However, the greatest challenge children often face is societal misunderstanding and stigma related to a disability whose cause lies in intergenerational struggle within family systems. The social construction of knowledge about FASD emerges from a society which values perfection and does not easily abide difference, thus contributing to a failure to recognize the unique and sacred nature of each person in the world. Children with FASD are at high risk of abuse, a lack of understanding regarding neurobehavioral problems and are particularly vulnerable to those who would take advantage. One of their greatest vulnerabilities is societal perception of who they are based on media representation of individuals with FASD as more likely to be engaged in criminal activity and a danger to self and others. More recently Sullivan (2009) focuses on underlying vulnerabilities and problems in life adaptations including "poor parental role modeling, disturbed development of trust and identity, patterns of avoidant coping behaviour, dysfunctional adolescent and adult relationships, and economic disadvantage" (p. 226). These concerns are realities for adults with FASD and indeed pose challenges because intervention aimed at ameliorating these concerns is time-intensive and costly. A small literature is beginning to appear that focuses on the quality of life of individuals living with FASD that may provide a fuller picture of what it means to live with FASD and how we can improve the quality of life for children, adolescents and adults (Stade et al. 2006; Grant et al., 2005).

\section{Service Delivery}

Services delivered to children in care are largely determined by the assigned caseworker with guidance from a casework supervisor and existing policies. The competence of the worker and their knowledge about FASD will influence their choice of services accessed for children. At present there are no formal measurements of the level of knowledge of caseworkers regarding FASD. In this project, all workers and caregivers within the pilot project received training specific to the standards. A total of 1932 training hours were logged over the duration of the 
two year project.

An Eco-Map for a child with FASD in care could include some of the following needs as determined by the caseworker: caregiving system (foster care, youth and child care network); adequate funding for support services and community involvement; youth justice network; education; medicalcareincluding access to otherhealth professions such as services offered by a psychologist, occupational therapy, physiotherapy, speech therapy; disability accommodations as required; transitional planning; and finally a social life which includes a connection to family if possible including immediate or extended family members. Concerns raised in the focus groups with foster parents were tensions regarding the issue of family visitation. Although it may be controversial to raise this concern, its identification within the practice standard identified as Home Visits is important in the interest of best practice.

The standard on Home Visits required caseworkers to complete a family visitation checklist and a family visitation plan. The rationale identified in the standards regarding this planning is that it is important to anticipate the potential benefits and detriments of familial contact. Dependent on the circumstances related to how the child came into care, the relationship between the family and the agency and ongoing protection concerns, it is recognized that this is a sensitive issue. A standard was identified to ensure that the child derived benefit from the visit as well as the family. It was also believed that structure around the visits was important including time, location and supports required. The concerns identified regarding the visits were that they affect the routine, attachments and bonds in the foster home. An internal conflict for children is that they do not want to leave their biological family, or their foster family. Another concern identified related to the grief children experienced after a family visit and that it sometimes took several days to re-establish a routine post family visitations. When families did not show up for scheduled visits this also created considerable distress for children.

It is important to recognize that visiting their children who have been removed from their care is often a painful experience for parents. There needs to be a sensitivity protocol established for interacting with biological families by the child welfare system. It is important for caseworkers to use caution in how information on alcohol consumption is gathered as this can trigger overwhelming guilt and grief responses that constitute a risk for women. Research on birth mothers of children with FASD unequivocally identifies the need for a woman centered approach in working with families (Poole and Greaves (2009) Badry, (2009); Boyd and Marcellus (2007), Poole (2003), Grant, Ernst, Streissguth and Stark,(2005) Greaves et al. (2002), Lesichner (2001) Rutman, Callahan, Lundquist, Jackson
\& Field (2000). Dell and Roberts (2005) suggest greater efforts be made on the part of professionals in terms of their knowledge and preparation to care for women who are dependent/misusing alcohol. In raising this issue it is important to consider how best to intervene with families and ameliorate these concerns. The seriousness of child protection concerns must not be minimized. However, utilizing an approach that does alienate families is also crucial. The disruption of the parent/child bond through the placement of children in care influences attachment and can lead to later problems in development (Alberta Children's Services Workforce Development and Gough, 2007). Wherever possible, these disruptions should be minimized. Providing connections and anchors for the child or young person in care to their community on a regular basis, in the presence or absence of their parent(s) can offer security to children and families. Neckoway, Brownlee and Castellan (2007) raised the question of Aboriginal parenting realities relevant to attachment theory and support the need for considering the role of extended family and kin. Perhaps healthy attachment between child and community may mediate some of the difficulties identified regarding family visitation.

If visits are perceived as disruptive this notion needs to be challenged as policy requires they occur unless they have been discontinued based on a court order such as permanent guardianship. Creative ways of involving children with the community such as monthly community days may be considered as a way to keep children connected to their relatives and identity in the absence of parents. Engaging in "community" visitation on a regular basis versus individual family members may offer consistent support to children, families and the community as a whole, and minimize the discourse of disruption around the process of visitation. Children with FASD need routine, structure and consistency. Addressing family visitation from a position that involves the community may be an important consideration for future practice. This approach supports the child, the family and the community to stay connected. Finally, this issue needs to be addressed from a holistic and ecological perspective with various stakeholders in order to bring resolution to these concerns while keeping children linked to their communities on a regular basis.

\section{FASD Practice Standards Research}

At the beginning of the implementation and evaluation of the FASD Practice Standards project, researchers and caseworkers believed that this was critical social science research that must be done. Awareness of increasing numbers of children in care with suspected or diagnosed incidence of FASD is a burgeoning concern. Our research employed both qualitative and quantitative methodologies. There were 33 children in the pilot group and 30 in the control group 
in relation to the casework standards. Changing casework practice required a paradigm shift and the implementation of a new model of practice was identified as an important phenomenon to study. Focus groups were held separately with caseworkers and foster parents to develop an understanding of the experience of this process. The underlying research question was whether the standards made a difference in outcomes for children in care with FASD.

One of the issues raised by the team developing and initiating the standards was the concept of what constitutes being a "good guardian" for children in care with FASD. It was recognized within the development of the standards that the guardianship of children with FASD was complex, and required additional intervention and support by caseworkers. The construct of improving care was worthy of attention in this research. The commitment to offering more casework hours for children at a multiplier of 1.5 was critical to the success of the project. Additional staff was hired in order to decrease caseloads and support increased time for the worker in terms of face to face contact, visitation to the foster home and completing required documentation.

The research included focus group discussions with staff and foster parents in the pilot region at the beginning, mid-point and end of pilot implementation as well as case reviews to determine the level of compliance with the practice standards. Second, for the purposes of hypothesis testing, a quasi-experimental matched comparison group design was utilized involving a pilot group that implemented the Practice Standards, and a comparison group, which did not. Children included in this study were matched based upon age, gender, child welfare status, number of years in foster care and diagnostic classification (i.e. diagnosed or suspected). An assumption was made that children identified for assessment of FASD should be included within the study as they were likely to receive benefit from being involved.

In creating the measurement instrument, decisions were made to monitor behaviors that were of concern to the caseworkers including, self-harm, drug and alcohol misuse, AWOL (absence without leave) and involvement in criminal activity. These concerns were previously identified in the research literature by Streissguth (1997) as secondary disabilities. In addition to designing instrumentation to measure these problems, along with time logs for case managers. Each form of risk behaviour demonstrated a downward trend over the duration of the implementation of the practice standards with the steepest decrease for criminal behaviour and drug use.

The casework standards were designed to offer early identification of FASD through screening and referral, planning for specific needs of children based on assessment/diagnosis, increased training on FASD for caseworkers, foster parents and community based program staff. Program standards were developed in foster care, kinship care, adoption, private guardianship, residential care, family preservation and youth mentoring services. Desired outcomes included increased placement stability, decreased incidences of behavioral problems and risk behaviors, as well as effective transition to adult services, including the completion of transitional plans to adulthood by age 16 .

To address the impact of the Practice Standards, this project examined three major outcomes within the Aboriginal unit: residential placement stability, quality of relationships with caregivers and teachers, and risk behaviours. There were no statistically significant differences in relation to gender, diagnostic classification and age between the Pilot (Lethbridge, Alberta and area) and Comparison groups (Calgary, Alberta). However, the Comparison group had a much higher proportion of PGO status cases than did the Pilot group. While the Pilot group experienced higher rates of placement change before and during implementation of the Practice Standards, there was a significant decrease in placement changes over time resulting in a convergence of mean placement changes over time. Consistent patterns of reduced risk behaviors and school absences were associated with the implementation of the Practice Standards. Despite a trend of decreasing respite care during the implementation phase, mean ratings of the perceived quality of relationships at home and school as well as self-reported placement satisfaction increased during the implementation of the Practice Standards.

\section{Diagnosis of FASD}

The issue of diagnosis of FASD emerged as an important phenomenon though the research based on information retrieved in focus groups. The origins of FASD emerge from the medical model (Jones and Smith, 1973; Lemoine, 1968). From a medical paradigm the issue of diagnosis is critical in terms of responding to an illness or chronic medical condition. It is important in relation to the FASD that child welfare and the medical profession work collaboratively in terms of assessment and diagnosis. It is not possible to engage in a discussion of diagnostic terminology in the context of this paper as this is an extensive topic and at times controversial.

Children with suspected or diagnosed FASD often come to the attention of child welfare due to the issues of substance misuse in their homes. The issue of how children became aware of their diagnosis of FASD came to our attention through the focus groups. Although both the child welfare and medical system support the importance of a diagnosis in mediating the concerns and needs for children with disabilities as a result of alcohol exposure in utero, some children experience grief when discovering they have received a diagnosis of FASD. In deconstructing this issue it is important to consider the implications of a diagnosis 
for a child. Some children reacted to this news by being offended, angry about being labeled and had difficulty understanding the meaning of the diagnosis.

A practice standard identified as Child and Family Awareness of FASD suggests that receiving a diagnosis will help children and families to receive support in dealing with barriers and problems they face. Foster parents indicated that not all children are ready to hear about the diagnosis and this information sometimes leads to negative changes and turmoil within the child. Children have also "shutdown" after hearing this information and some experience depression. The information provided to biological families must be presented by the caseworker and it was acknowledged that this was a challenging task. It was discovered that children receive information about a diagnosis of FASD from several different sources including medical professionals, caseworkers and foster parents. Children are challenged to meaningfully interpret what this diagnosis means and it was suggested as a result of this research that a protocol for disclosure of FASD to children must be developed in child welfare practice.

There are many issues that require further exploration as a result of reviewing the data generated from both qualitative and quantitative data. It is important to note that despite the challenges of implementing enhanced standards of care for children with FASD, the commitment to this project from a regional perspective was profound. Further, it must be stated that enhanced supports for children with FASD are important. Our research supports the case that some specialization in the delivery of child welfare services is required for this population. It must be noted that this evaluation contributed to greater awareness of the many activities which caseworkers and foster parents engage in to support children with FASD. It also provided an opportunity for these groups to reflect on their experiences in caring for children with complex needs, and offers important insight that can inform future practice.

\section{Key Findings}

- While the Pilot group experienced higher rates of placement change before and during implementation of the Practice Standards, there was a significant decrease in placement changes over time resulting in a convergence of mean placement changes over time.

- Consistent patterns of reduced risk behaviors and school absences were associated with the implementation of the Practice Standards.

- Specific training in FASD supports caseworkers and foster parents in working with children and in meeting their needs more effectively. Training opportunities are valued and more specialized training is required.

- Caseworkers and foster parents were invested in the application of the standards, valued the experience, and the opportunity to reflect on their practice.

- Family visitation is a major site of conflict and tension, and considered to be the biggest factor in the "disruption" of the life of the child by foster families.

- The application of standards appeared to minimize other life disruptions such as school absences, behavioral difficulties, and change of placement.

- The diagnosis of FASD impacts the emotional well being of the child and leads to a key recommendation that a protocol for disclosure of FASD to children and families should be jointly established between diagnostic clinics and child welfare agencies.

- The use of respite should be negotiated with foster families.

- The standards state that there should be a minimum of two children with FASD in a home, and this is a source of conflict for some foster families.

\section{Conclusions}

The topic ofFAS/FASD is phenomenon of great interest to many sectors in the professional world such as medical doctors, social workers, nurses, psychologists, occupational therapists, counselors, youth and childcare workers, foster parents, community and government professionals. The FASD Practice Standards research that took place between 2003-2005 yielded valuable information in regards to child welfare practice for children with FASD in care. As a result of this research, awareness of the complexity of children in care with FASD was examined and the final recommendations of this study are important to highlight in conclusion.

Training on the complexity of fostering and engaging in casework for children diagnosed with FASD should be ongoing with particular focus on different issues at different ages (early childhood/pre-school, school age, and adolescence, young adulthood). Given the complex needs of children with FASD a recommendation was made that no more than two children with FASD be placed in one foster home, to support the long term stability of the placement. However, in the case of sibling groups this may not be in the children's best interest and may require a flexible response. It is important to take a long term perspective if children are in permanent care and there needs to be awareness and consideration regarding the ability of foster parents to effectively meet the needs of more than two children with FASD.

The disclosure of FASD to a child has huge implications in their lives. The child must deal with being diagnosed with a disability, the history of their family, the societal biases which are inherent in a diagnosis, and the child's own 
concern about what this means. One standard addresses family awareness of FASD, but there is not consensus on how much information needs to be provided to a child. The impact of a diagnosis requires a specific set of supports and guidelines that address the experiences of trauma and grief which can emerge subsequent to learning this information. A recommendation was also made that Children's Services undertake a dialogue aimed at addressing the issue of family contact and visitation from a holistic, ecological perspective to diminish its emergence as a serious site of conflict for everyone involved.

A final recommendation made in 2005 at the conclusion of this project was that the FASD Practice Standards be implemented in other regions. This research demonstrated positive results for children, families, caseworkers and foster parents. Focusing the spotlight on specialized practice in FASD and systemic responses to this issue highlighted a worthwhile effort on the part of Alberta Children's Services to deliver services in a new way. The research also highlighted areas of concern such as conflict over the issue of visitation, a topic that merits further work. A common concern related to best practice for children with FASD emerged as a result of this project and dialogue was generated about the need to practice differently by offering increased hours of service. The benefit of offering specific training assured that everyone involved in the project received the same information, thus increasing the possibility of a consistent response to children.

Finally, in December, 2009, Alberta Children's Services announced that the FASD Practice Standards project would be expanded to four Child and Family Service Authorities based on the success of the earlier pilot. The four regions include the initial pilot site in Region 1 (Southern Alberta), Region 7 which encompasses a very large geographic area from Lloydminster to Jasper, Region 9-Fort McMurray and Region 10- Land Based Métis Settlements. The inception and initiation of new models of practice in the delivery of child welfare services is a worthwhile endeavour. It is anticipated that growing awareness of the complex needs of children with FASD and responses grounded in innovative practice will better serve children.

\section{References}

Alberta children's Services Workforce Development \& Gough, P. (2007). Supporting healthy attachment: An overview for child welfare practitioners. CECW Information Sheet \#56E, Retrieved online November 8 from http://www.cecw-cepb. $\underline{\mathrm{ca} /}$.

Armstrong, E. (2003). Conceiving risk, bearing responsibility: Fetal alcohol syndrome \& the diagnosis of moral disorder. Baltimore: John Hopkins University Press.

Armstrong, E. \& Abel, E. (2000). Fetal Alcohol Syndrome: The origins of moral panic. Alcohol and Alcoholism 35, (3) 276282.

Aronson, M. (1997). Children of alcoholic mothers: results from Goteborg, Sweden. In. Streissguth, A.P. and Kanter, J. (Ed.), The challenge of fetal alcohol syndrome: overcoming secondary disabilities (pp. 15-24). Seattle: University of Washington Press.

Badry, D. (2009). Becoming a Birth Mother of a Child with Fetal Alcohol Syndrome. Unpublished Dissertation, Graduate Division of Educational Research, University of Calgary.

Badry, D. \& Pelech, W. and Norman (2005). Fetal Alcohol Spectrum Disorder Practice Standards Evaluation Project Final Report. Alberta Children's Services.

Barr, H., \& Streissguth.A. (February 2001). Identifying maternal self-reported alcohol use associated with Fetal Alcohol Spectrum Disorder. Alcoholism: Clinical and Experimental Research, 25(2), 283-287.

Berube, M. (2005). Modern lepers: From person to political, prevention of FASD through working with marginalized women. Unpublished master's case-study, Faculty of Social Work, University of Calgary, Calgary, Alberta.

Boyd, S. (2004). From Witches to Crack Moms: Women, Drug Law and Policy. Durham, North Carolina: Carolina Academic Press.

Boyd, S. \& Marcellus., L. (2007). With child: substance use during pregnancy. A woman centred approach. Halifax: Fernwood Publishing.

Chudley, Albert E., et al. "Fetal Alcohol Spectrum Disorder: Canadian guidelines for diagnosis." Canadian Medical Association Journal, 172.5 Suppl (2005): S1-S21.

Clarren, S.K. and Lutke, J. Building clinical capacity for Fetal Alcohol Spectrum Disorder diagnoses in Western and Northern Canada. Canadian Journal of Clinical Pharmacology (2009)15, 2: e223-e237.

Dell, C. \& Roberts, G. (2005). Research Update: Alcohol use and pregnancy: An important Canadian public health and social issue. Public Health Agency of Canada.

Fetal Alcohol Spectrum Disorder Practice Standards. (2003). Southwest Alberta Child and Family Services, Alberta Children's Services.

Ferguson, L. (1997). Deconstructing Fetal Alcohol Syndrome: A critical inquiry into the discourse around alcohol, women, ethnicity, Aboriginals and disease. (Unpublished Masters thesis, Carleton University, Ottawa, Ontario).

Fuchs, D., Burnside, L., Marchenski, S. and Mudry, A. (2005). Children with disabilities receiving services from child welfare agencies in Manitoba. Winnipeg, MB: Centre of Excellence for Child Welfare. Retrieved online from http:// www.cecw-cepb.ca.

Gearing, R., McNeill, T. \& Lozier, F. (2005). Father involvement and fetal alcohol spectrum disorder: developing best practices. Journal of FAS International, Article 3e14, Retrieved online October 12, 2007 from http://www.motherisk.org/FAR/ 
econtent_archives.jsp?archive id=5.

Gough, P. (2006). Alberta's child welfare system. CECW Information Sheet \#46E. Retrieved October 20, 2009, 2009, from www.cecw-cepb.ca.

Gough, P., Trocme', N., Brown, I., Knoke, D. \& Blackstock, C. (2006). Pathway to the overrepresentation of Aboriginal children in care. CECW Information Sheet \#23E. Retrieved October 20, 2009, 2009, from www.cecw-cepb.ca.

Grant, T., Ernst, C., Streissguth, A., \& Stark, K. (2005). Preventing alcohol and drug exposed births in Washington State: intervention findings from three parent-child assistance program sites [Electronic Version]. The American Journal of Drug and Alcohol Abuse, 31, 471-490. Retrieved March 16, 2007.

Hutson, J. (2004). "Meconium Fatty Acid Ethyl Esters and Predictions of Fetal Alcohol Effects." Journal of Fetal Alcohol Syndrome International, 4: e15-e18.

Jones, K. L., Smith, D., Ulleland, C., \& Streissguth, A. (1973). Patter of malformation in offspring of chronic alcoholic mothers. The Lancet, 1267-1271.

Lemoine, P. (2003). The history of alcoholic fetopathies. Journal of FAS International,4, Article 1e2, Retrieved online October 12, 2007 from http://www.motherisk.org/FAR/econtent archives.jsp? archive id=5.

Marsh, J., \& Dingcai, C. (2005). Parents in substance abuse treatment: implications for child welfare practice [Electronic Version]. Children and Youth Services Review, 27, 1259-1278.

Neckoway, R., Brownlee, K. \& Castellan, B. (2007). Is attachment theory consistent with aboriginal parenting realities? First Peoples Child \& Family Review, 3(2), 56-64.

O’Malley, K. (Ed.) (2009). ADHD and fetal alcohol spectrum disorders (FASD). New York: Nova Science Publishers.

Poole, N. (2003). Mother and child reunion: Preventing fetal alcohol spectrum disorder by promoting women's health. Vancouver, BC: Centre for Excellence in Women's Health.

Roy, C., Black, T., Trocmé, N., MacLaurin, B. \& Fallon, B. (2005). Child neglect in Canada. CECW Information Sheet \#27E. Retrieved online November 9, 2009 from http://www. cecw-cepb.ca.
Stade, B. C., Stevens, B., Ungar, W., Beyene,J. \& Koren, G. (2006) "Health-Related Quality of Life of Canadian Children and Youth Prenatally Exposed to Alcohol." Health and Quality of Life Outcomes 4.81. Retrieved online November 9, 2009 from http://www.pubmedcentral.nih.gov/articlerender. fcgi?artid=1617087.

Stratton, K., Howe, C. \& Battaglia, F. (Eds.) (1996). Fetal alcohol syndrome: Diagnosis, epidemiology, prevention and treatment. Washington, D.C.:Institute of Medicine, National Academy Press.

Streissguth, A. (1997). Fetal alcohol syndrome: A guide for families and communities. Baltimore, MD: Brookes.

Streissguth, A.P. and Kanter, J. (Ed.), (1997). The challenge of fetal alcohol syndrome: overcoming secondary disabilities (pp. 15-24). Seattle: University of Washington Press.

Streissguth, A.P. \& O’Malley, K. (2001). Neuropsychiatric implications and long-term consequences of fetal alcohol spectrum disorders. Retrieved online from MEDLINE. ISSN: 1084-3612.

Sullivan, A. (2009). "Fetal alcohol spectrum disorders (FASD) in the adult: Vulnerability, disability, or diagnosis - a psychodynamic perspective." In O'Malley, K. (Ed.). ADHD and Fetal Alcohol Spectrum Disorders (FASD). New York: Nova Science Publishers. (pp. 215-245).

Tait, C. (2000a). A study of the service needs of pregnant addicted women in Manitoba. Manitoba Health: Prairie Women's Health Centre for Excellence.

Tait, C. (2000b). Aboriginal identity and the construction of Fetal Alcohol Syndrome. Montreal, Quebec: Proceedings of the Advanced Study Institute: The Mental Health of Indigenous Peoples. Culture and Mental Health Research Unit Report No. 10. Retrieved February 2, 2003 from http://www.mcgill. ca/psychiatry/transcultural/pdf/Report10.pdf.

Tait, C. (2003a). Fetal Alcohol Syndrome among Aboriginal people in Canada: Review and analysis of the intergenerational links to residential schools. Montreal, Quebec: The Baronial Healing Foundation.

Tait, C. (2003b). The tip of the iceberg": The "making” of fetal alcohol syndrome in Canada. Doctoral Dissertation, McGill University, Montreal, Quebec. Retrieved October 14, 2007 from http://www.mcgill.ca/files/namhr/Tait-2003.pdf. 